\title{
A STRATEGY FOR LINKING HIPPARCOS TO AN EXTRA-GALACTIC REFERENCE FRAME
}

\author{
A.N. Argue ${ }^{1}$ \\ Institute of Astronomy, \\ University of Cambridge, \\ Madingley Road, Cambridge CB3 0HA, UK.
}

\begin{abstract}
The strategy for linking the HIPPARCOS Catalogue to extragalactic objects originally devised for the geostationary orbit, is no longer adequate for the elliptical orbit to which the satellite is now confined, but useful improvements in the rotation matrix may be obtained by incorporating proper motions from certain other catalogues and from ground based absolute proper motions.
\end{abstract}

\section{Introduction}

The locking of HIPPARCOS out of its geostationary orbit has created the urgent need for a drastic reappraisal of the strategy for the extragalactic link.

At the time of writing, there is an estimated $50 \%$ probability of the life time exceeding 18 months, but this is highly uncertain since it not estabilshed that it will be possible to reactivate the payload following the periods of prolonged eclipse. The following sigmas as a function of life expectancy have been predicted from simulations by Lindegren:

Life time position parallax proper motion

$$
\begin{array}{ccc}
\sigma_{\alpha, \delta} & \sigma_{\pi} & \sigma_{\mu}
\end{array}
$$

$\begin{array}{llll}1.5 y r & 0^{\prime \prime} .006 & 0^{\prime \prime} .0060 & 0^{\prime \prime} .008 y r^{-1} \\ 2.0 & 0^{\prime \prime} .003 & 0^{\prime \prime} .0045 & 0^{\prime \prime} .006 y r^{-1} \\ 2.5 & 0^{\prime \prime} .003 & 0^{\prime \prime} .0035 & 0^{\prime \prime} .005 y r^{-1}\end{array}$

1JANET: ANA1@PHX.CAM.AC.UK 
(For the geostationary mission originally planned, the duration would have been $2.5 \mathrm{yr}$ and each $\sigma=0^{\prime \prime} .002$.) The estimates that follow here are based on a 2.5yr life time.

If a reconstitution of the celestial sphere can be performed successfully from the satellite data, there will result a system of positions, trigonometric parallaxes and proper motions for about 100,000 stars on a homogeneous, rigid instrumental system. The zero points for position and proper motions will be arbitrary in the first instance but will be aligned to FK5 as an off-line stage in the reduction. Lastly, the HIPPARCOS Catalogue will be aligned to the VLBI frame.

\section{The Positional Frame}

The positional frame will be greatly superior to any optical frame we now possess. In comparing it to FK5, it will be possible to smooth out small zonal errors known to exist in FK5 [1]. Then, linked to VLBI, there will be, for the first time, a homogeneous frame unified for optical and radio astrometry. This is urgently needed for the accurate correlation of optical and radio features in sources.

\section{The Inertial Frame}

For the solid body rotation for the frame, however, the rescue mission will not give any significant strengthening of FK5 beyond what is known already, when the methods are confined to those envisaged by the FAST/NDAC link for the original mission [2] namely, using radio stars and Hubble Space Telescope $[3,11]$. This is because both the numbers of objects and the predicted accuracy have become insufficient (the results now to be expected are given in the first line of Table 2). Although smaller than the $\sigma_{\mu}$ for one star $\left(0^{\prime \prime} .005 \mathrm{yr}^{-} 1\right)$, nevertheless much better values are needed for dealing with groups of stars, for instance in studies of galactic rotation. We shall show that improvements may be obtained by incorporating proper motions from other catalogues.

\section{Proper Motions from other Catalogues}

We propose that, following the transformation of the HIPPARCOS proper motions to FK5, the catalogues listed in Table 1 be then transformed to this HIPPARCOSFK5 system. This could be done accurately because of the large overlap, as specified by the numbers in the third column. The FAST/NDAC procedures are then applied using these transformed catalogue proper motions in place of the HIPPARCOS instrumental values as originally planned for the geostationary mission. The likely numbers of stars in the FAST/NDAC proposals, selected for use with Space Tele- 
scope (HST) and radio stars respectively, are given in the fourth and fifth columns of Table 1. The numbers given for CAMC/Bordeaux are actual counts; the remaining numbers are estimates based on the various catalogue limits. For $\mathrm{AC}$, the magnitude cut-off is assumed to be $B=11^{m}$ and for IRS+NPZT and ACRS, $V=10^{m} .5$. The declination limit for CAMC is $-45^{\circ}$. The last five lines refer to "absolute proper motion" catalogues discussed in Section 7.

\section{Accuracy of Rotation Link}

We have used the simulations by Froeschlé and Kovalevsky [4] to estimate the uncertainty $\sigma \mathrm{R}^{\prime}$ in the time derivative $\mathrm{R}^{\prime}$ of the fixed matrix rotation $\mathrm{R}$ for the first five catalogues in Table 1 .

The results are in Table 2. Again we have used the limits of the various catalogues to estimate the numbers of objects, except for CAMC/Bordeaux: in the cases of IRS+NPZT and ACRS these estimates are very imprecise because we have not attempted to identify which particular stars are involved. For AC and CAMC/Bordeaux we have assumed 40 radio stars, this being the number likely to have accurately measured radio proper motions available at the end of the mission. For the VLBI accuracy we have assumed $0^{\prime \prime} .002$.

\section{Conclusion}

For the radio star link, AC and CAMC/Bordeaux in particular shew useful improvements over the rescue mission in the top line of Table 2. Indeed a straight mean of $\mathrm{AC}$ and $\mathrm{CAMC} /$ Bordeaux would give results very close to those expected for the original mission $\left(0^{\prime \prime} .0020 \mathrm{yr}^{-1}\right.$ for HST and $0^{\prime \prime} .0008 \mathrm{yr}^{-1}$ for radio stars).

\section{Ground based Optical Proper Motions}

The last four lines in Table 1 refer to surveys that are directly tied to extragalactic objects. The values anticipated for $\sigma \mathrm{R}^{\prime}$ are (with the corresponding number of radio sources in parenthesis):

$\begin{array}{ll}\text { Lick/Yale } & 0^{\prime \prime} .0002 y r^{-1} \\ \text { Potsdam } & 0^{\prime \prime} .0014 \\ \text { Kiev } & 0^{\prime \prime} .0007 \\ \text { Bonn } & 0^{\prime \prime} .0003 \\ \text { Hamburg/La } & 0^{\prime \prime} .0005 \\ \text { Palma/CSIRO } & \end{array}$


Table 1: Surveys of Proper Motion: the third column gives the overlap between the catalogue and HIPPARCOS, the fourth and fifth the number of HIPPARCOS stars from the original proposals. Not all of the radio stars may have radio astrometry available at the end of the mission.

The mean errors for AC are from Brosche \& Geffert [5]; ACRS (AC Reference Star Catalogue) from Corbin \& Urban [8]; CAMC from Morrison et al. [6]; PPM from Röser and Bastian; CPC2 from de Vegt et al. [9]; Kiev from Yatsenko et al. [10] and the remainder from The HIPPARCOS Mission II [2].

\begin{tabular}{lcrrr}
\hline Catalogue & $\begin{array}{c}\text { Mean Error } \alpha, \delta \\
\left(\text { arcs.yr } r^{-1}\right)\end{array}$ & $\begin{array}{r}\text { No. in } \\
\text { Mission }\end{array}$ & $\begin{array}{r}\text { No. in } \\
\text { HST Link }\end{array}$ & $\begin{array}{r}\text { No. in } \\
\text { Radio Stars }\end{array}$ \\
\hline HIPPARCOS & $0^{\prime \prime} .005$ & 100,000 & 147 & 157 \\
\hline AC & $0^{\prime \prime} .003$ & $\sim 90,000$ & 90 & 142 \\
PPM+CPC2 & $0^{\prime \prime} .004$ & $\sim 55,000$ & 92 & $\sim 60$ \\
IRS+NPZT & $0^{\prime \prime} .005$ & $\sim 38,000$ & $\sim 30$ & $\sim 60$ \\
ACRS & $0^{\prime \prime} .004$ & $\sim 90,000$ & $\sim 80$ & $\sim 142$ \\
CAMC/Bordeaux & $0^{\prime \prime} .003$ & $\sim 38,000$ & 77 & $40 / 61$ \\
\hline & & & & \\
Lick/Yale & $0^{\prime \prime} .005$ & 20,000 & & \\
Potsdam & $0^{\prime \prime} .003$ & 120 & & \\
Kiev & $0^{\prime \prime} .003$ & 340 & & \\
Bonn & $0^{\prime \prime} .002$ & 200 & & \\
Hamburg/La Palma/ & $0^{\prime \prime} .004$ & 300 & & \\
CSIRO & & & & \\
\hline
\end{tabular}


Table 2: Uncertainty $\sigma \mathrm{R}^{\prime}$ in the frame rotation $\mathrm{R}^{\prime}$ for six surveys of Table 1. In parenthesis: the number of radio sources assumed.

\begin{tabular}{|c|c|c|c|c|}
\hline Catalogue & $\begin{array}{l}\sigma \mathrm{R}^{\prime} \\
\text { HST Link }\end{array}$ & & $\begin{array}{l}\sigma \mathrm{R}^{\prime} \\
\text { Radio Stars }\end{array}$ & \\
\hline HIPPARCOS & $0^{\prime \prime} .0040 y r^{-1}$ & (78) & $0^{\prime \prime} .0021 y r^{-1}$ & $(40)$ \\
\hline $\mathrm{AC}$ & $0^{\prime \prime} .0028 y r^{-1}$ & (64) & $0^{\prime \prime} .0014 y r^{-1}$ & $(40)$ \\
\hline $\mathrm{PPM}+\mathrm{CPC} 2$ & $0^{\prime \prime} .0036$ & (60) & $0^{\prime \prime} .0020$ & (30) \\
\hline IRS+NPZT & $0^{\prime \prime} .0061$ & (25) & $0^{\prime \prime} .0034$ & (15) \\
\hline ACRS & $0^{\prime \prime} .0036$ & (60) & $0^{\prime \prime} .0022$ & (36) \\
\hline CAMC/Bordeaux & $0^{\prime \prime} .0027$ & (77) & $0^{\prime \prime} .0014$ & $(40)$ \\
\hline
\end{tabular}

Again, $\sigma \mathrm{R}^{\prime}$ is significantly better than the proper motion of an individual star as measured by the rescue mission. These absolute proper motion surveys will provide a valuable supplement to the methods of Section 5 . The original plan was to relegate these ground based methods to an inferior status of 'private projects' and use only the FAST/NDAC link for the HIPPARCOS Catalogue. Now it is urged that these methods have a much more vital rôle to play. 


\section{References}

1. L. V.Morrison, P. Gibbs, L. Helmer, C. Fabricius, O. Einicke, Y. Réquième and M. Rapaport, 1990. Evidence of systematic errors in FK5. In 'Fundamentals of Astrometry', Proc. IAU Coll. 100, H. Eichhorn (ed.), in preparation.

2. A.N.Argue, 1989. The Link to Extragalactic Objects. In 'The HIPPARCOS Mission II', M. A. C. Perryman and C. Turon (eds.), ESA SP-1111 p. 199.

3. P. D. Hemenway, G.F. Benedict, W. H. Jefferys, P. J. Shelus and R. L. Duncombe, 1988. The Extragalactic Link. Operational Preparations for the Hubble Space Telescope Observations. In 'HIPPARCOS Scientific Aspects of the Input Catalogue Preparation', J. Torra and C. Turon (eds.), CIRIT, p. 461.

4. M. Froeschlé and J. Kovalevsky, 1982. The Connection of a Catalogue of Stars with an Extragalactic Reference Frame, Astron. Astrophys. 116, 89.

5. P. Brosche and M. Geffert, 1988. In 'Mapping the Sky', Proc. IAU Symp. 133, S. Débarbat et al. (eds.), p. 403.

6. L. V. Morrison, L. Helmer and L. Quijano, 1988. Mapping the Sky with the Carlsberg Automatic Meridian Circle, Ibid. p. 369.

7. S. Röser and U. Bastian, 1989. Compilation of the PPM Catalogue. In 'Star Catalogues: A Centennial Tribute to A. N. Vyssotsky', A. G. Philip and A. R. Upgren (eds.), p. 31.

8. T.E. Corbin and S. E. Urban, 1898. Proper Motions of the Northern Astrographic Catalogue Reference Stars, Ibid. p. 59.

9. Ch.de Vegt, N.Zacharias, C.A. Murray and M. J. Penston, 1989. A Progress Report on the Second Cape Astrographic Catalog, Ibid. p. 45.

10. A. I. Yatsenko, S. P. Rybka and R.-D.Scholz, 1987. The Connection of the HIPPARCOS reference system to extragalactic objects by photographic astrometry, Astron. Nachr. 308, 319.

11. F. Mignard, 1989. HIPPARCOS and Reference Systems. In this symposium.

\section{Discussion}

STRAND: It seems the value of 0.0002 arcsec for the Lick/Yale program is small. Can you explain this?

ARGUE: Yes, by the very large overlap between the Lick/Yale and the HIPPARCos programmes.

YATSKIv: Why didn't you take into account the proper motion data from the Pulkovo program (i.e., the Tashkent, Kiev, and Pulkovo catalogues)?

ARGUE: I hope to receive more information on this programme at the joint meeting of IAU Commission 24 Working Group on Radio-Optical Objects and the HIPPARCos INCA WG 2130 , to be held here tomorrow. 